\title{
Justiz-Initiative: Eckpunkte eines Gegenentwurfs und Ausblick
}

Die Schweizerische Vereinigung der Richterinnen und Richter (SVR-ASM) lehnt die Justiz-Initiative ab. Mit Blick auf die Schwächen des heutigen Wahlsystems fordert die SVR-ASM jedoch einen Gegenentwurf. Am 5. November 2020 hat sie nach einer breiten internen Vernehmlassung Eckpunkte eines solchen Gegenentwurfes präsentiert und damit erfreulicherweise beim Parlament Gehör gefunden.

Beitragsart: Kolumne SVR

Zitiervorschlag: Patrick Guidon, Justiz-Initiative: Eckpunkte eines Gegenentwurfs und Ausblick, in: «Justice - Justiz - Giustizia» 2020/4 


\section{Inhaltsübersicht}

1. Einleitung

2. Schwächen des heutigen Systems

2.1. Periodische Wiederwahl

2.2. Mandatssteuern

2.3. Auswahlverfahren

3. Lösungen

3.1. Einführung der einmaligen Wahl

3.2. Abschaffung / Ersatz der Mandatssteuern

3.3. Professionalisierung des Auswahlverfahrens

4. Konkrete Eckpunkte eines Gegenvorschlags

4.1. Amtsdauer: Vorschlag für eine Änderung von Art. 145 BV

4.2. Auswahlverfahren: Vorschlag für eine Änderung von Art. 168 BV

4.3. Verbot der Mandatssteuer

4.4. Weitere Hinweise

5. Ausblick

\section{Einleitung ${ }^{1}$}

[1] Der Bundesrat hat am 19. August 2020 entschieden, dem Parlament die Ablehnung der Volksinitiative zur «Bestimmung der Bundesrichterinnen und Bundesrichter im Losverfahren (JustizInitiative)» ohne Gegenentwurf und Gegenvorschlag zu beantragen. ${ }^{2}$ Auch die Schweizerische Vereinigung der Richterinnen und Richter (SVR-ASM) lehnt die Justiz-Initiative ab, weil sie die bestehenden Probleme nicht in überzeugender Weise zu lösen vermag. Bedenken weckt insbesondere das mit der Initiative vorgeschlagene Losverfahren, welches die Bedeutung der demokratischen Legitimation von Richterinnen und Richtern durch eine Parlamentswahl ausblendet. ${ }^{3}$

[2] Allerdings macht die Justiz-Initiative zu Recht auf verschiedene Schwächen des heutigen Systems (nachfolgend Kap. 2) aufmerksam. Der Bundesrat hat es bedauerlicherweise ohne nähere Begründung abgelehnt, im Rahmen eines Gegenentwurfs diesbezügliche Lösungen vorzuschlagen. Die SVR-ASM hat deshalb an das Parlament appelliert, sich dieser wichtigen Aufgabe anzunehmen ${ }^{4}$ und der Kommission für Rechtsfragen des Nationalrates sowie der Öffentlichkeit am 5. November 2020 Lösungen (Kap. 3) und konkrete Eckpunkte eines solchen Gegenentwurfs (Kap. 4) präsentiert. ${ }^{5}$

1 Die folgenden Ausführungen geben das Positionspapier «Eckpunkte eines Gegenentwurfs zur Justiz-Initiative» der SVR-ASM vom 5. November 2020 wieder (abrufbar unter https://www.svr-asm.ch/de/index_htm_files/ 2020-11-05\%20Gegenentwurf\%20zur\%20Justiz-Initiative\%20d.pdf [deutsch]), https://www.svr-asm.ch/fr/index_ htm files/2020-11-05\%20Gegenentwurf\%20zur\%20Justiz-Initiative\%20f.pdf [französisch], https://www.svr-asm. ch/it/index_htm_files/2020-11-05\%20Gegenentwurf\%20zur\%20Justiz-Initiative\%20i.pdf [italienisch]). Das Positionspapier ist ein Gemeinschaftswerk des Vorstandes unter Einbezug sämtlicher Mitglieder. Entsprechend beansprucht der Autor nicht die (alleinige) geistige Urheberschaft hinsichtlich des nachstehend Gesagten.

2 Botschaft des Bundesrates vom 19. August 2020 zur Volksinitiative «Bestimmung der Bundesrichterinnen und Bundesrichter im Losverfahren (Justiz-Initiative)», BB1 20206821.

3 Vgl. hierzu die Medienmitteilungen der SVR-ASM vom 26. August 2019, «Justiz-Initiative: SVR-ASM fordert Gegenentwurf», (abrufbar unter https://www.svr-asm.ch/de/index_htm_files/2019-08-26\%20Medienmitteilung\% 20SVR-ASM\%20Justiz-Initiative.pdf) und vom 28. August 2020, «Justiz-Initiative: Es braucht einen Gegenentwurf», (abrufbar unter https://www.svr-asm.ch/de/index_htm_files/2020-08-28\%20Medienmitteilung\% 20SVR-ASM\%20Justiz-Initiative\%20-\%20Es\%20braucht\%20einen\%20Gegenentwurf.pdf).

4 Medienmitteilungen der SVR-ASM vom 28. August 2020 (Fn. 3).

5 Vgl. hierzu das in Fn. 1 erwähnte Positionspapier. 


\section{Schwächen des heutigen Systems}

\subsection{Periodische Wiederwahl}

[3] Die periodischen Wiederwahlen erhöhen das Risiko von Druckversuchen auf Richterinnen und Richter. Allein schon die theoretische Möglichkeit einer Nichtwiederwahl gefährdet die Unabhängigkeit der Richterinnen und Richter, denn damit besteht die Gefahr oder zumindest der Anschein, dass sachfremde Kriterien in die Entscheidfindung einfliessen könnten. Nach aussen entsteht zudem der Eindruck von Abhängigkeit vom Wahlorgan. Die Groupe d'Etats contre la corruption (GRECO) des Europarates hat das System der periodischen Wiederwahl wiederholt als Beeinträchtigung der richterlichen Unabhängigkeit kritisiert. ${ }^{6}$

\subsection{Mandatssteuern}

[4] Dass Richterinnen und Richter, die für ihre Wahl bzw. Wiederwahl auf die Unterstützung ihrer jeweiligen Partei angewiesen sind, dieser jährlich wiederkehrende Mandatssteuern in teils beträchtlicher Höhe ${ }^{7}$ leisten (müssen), erweckt in der Bevölkerung den Eindruck eines Abhängigkeitsverhältnisses zwischen Parteien und Richterschaft respektive, dass das Amt käuflich ist. ${ }^{8}$ Dieser Anschein allein beeinträchtigt das Vertrauen in die verfassungsmässig ${ }^{9}$ garantierte Unabhängigkeit der Gerichte, selbst wenn die Rechtsfindung im Einzelfall dadurch nicht beeinflusst wird. Im Unterschied zu politischen Mandatsträgerinnen und -trägern der Exekutive und Legislative, bei denen die Partei überdies Wahlwerbung leistet, gibt es auch keine sachliche und vermittelbare Rechtfertigung für diese Beiträge. Die Mandatssteuer stösst im Übrigen auch international auf Kritik. ${ }^{10}$

\subsection{Auswahlverfahren}

[5] Das heutige Auswahlverfahren über die Fraktionen und die Gerichtskommission schränkt die Auswahl qualifizierter Kandidierender ein, indem parteipolitisch ungebundene Kandidaturen faktisch ausgeschlossen werden. Ferner kann der Eindruck entstehen, dass nicht primär die fachlich und persönlich bestqualifizierten Personen zur Wahl vorgeschlagen werden, sondern die politisch ausgewogene Zusammensetzung des Gesamtgerichts bzw. entsprechende Absprachen der Fraktionen vorrangig sind. Das Argument der demokratisch legitimierten Ausgewogenheit der Zusammensetzung des Gerichts hat schliesslich wesentlich an Überzeugungskraft verloren, weil der Parteibeitritt sich zunehmend daran orientiert, wo die besten Wahlchancen bestehen.

6 Vgl. dazu Ziff. 101 und Empfehlung Nr. vi (iii) des Evaluationsberichts des GRECO vom 2. Dezember 2016 (abrufbar unter https://rm.coe.int/CoERMPublicCommonSearchServices/DisplayDCTMContent?documentId= 09000016806fced9) und Rz. 58 f. und 62 des Konformitätsberichts des GRECO vom 22. März 2019 (abrufbar unter https://rm.coe.int/vierte-evaluationsrunde- pravention-von-korruption-bei-mitgliedern-von-/168094e85f).

7 Siehe zur Höhe der Beiträge Giuliano Racioppi, Die moderne «Paulette»: Mandatssteuern von Richterinnen und Richtern, in: «Justice - Justiz - Giustizia» 2017/3.

8 Stellungnahme der Ethikkommission vom 27. März 2019, Mandatssteuern: Auch berufsethisch problematisch?, S. 5 (abrufbar unter https://www.svr-asm.ch/de/index_htm_files/Mandatssteuern.pdf).

9 Art. 30 Abs. 1 und Art. 191c BV.

10 Vgl. dazu Rz. 55 und 60 des Konformitätsberichts der GRECO vom 22. März 2019 (Fn. 6). 


\section{Lösungen}

\subsection{Einführung der einmaligen Wahl}

[6] Mit dieser im Kanton Freiburg ${ }^{11}$ bereits geltenden Lösung kann Druckversuchen via Wiederwahlen zuverlässig begegnet werden. Zu klären ist, ob die Wahl bis zum ordentlichen AHV-Alter oder allenfalls bis zu einem festgelegten Alter erfolgen soll. Als Korrektiv zur langen Amtsdauer ist eine Regelung zur Abberufung bei schwerer Pflichtverletzung oder Amtsunfähigkeit vorzusehen.

\subsection{Abschaffung / Ersatz der Mandatssteuern}

[7] Mit der Abschaffung der Mandatssteuern ${ }^{12}$ kann ein deutliches Zeichen für die Unabhängigkeit gesetzt werden. Sollte für die Parteien die finanzielle Unterstützung durch die Mandatsabgaben unverzichtbar sein, wären verschiedene Lösungen denkbar. So könnten beispielsweise die bereits heute gesetzlich verankerten Fraktionsbeiträge ${ }^{13}$ erhöht werden, um die entstehenden Ausfälle zu kompensieren.

\subsection{Professionalisierung des Auswahlverfahrens}

[8] Das Auswahlverfahren muss professionalisiert und so weit wie möglich objektiviert werden. Hierfür müssen insbesondere die Auswahlkriterien für die Richterinnen und Richter konkretisiert werden. Dabei wären nebst Fachkompetenz und richterlicher Erfahrung zwingend auch die Sozialkompetenz und Lebenserfahrung zu nennen. Weitere Aspekte sind die geschlechtliche und sprachliche Ausgewogenheit. Schliesslich ist bei der Wahl auch auf eine ausgewogene $\mathrm{Zu}$ sammensetzung des Gerichts in weltanschaulicher Hinsicht zu achten, unabhängig davon, ob die Kandidierenden Mitglied einer politischen Partei sind oder nicht.

[9] Nach Auffassung des Vorstands und einer überwiegenden Mehrheit der Mitglieder der SVRASM bietet sich für die notwendige Professionalisierung die Schaffung einer unabhängigen Fachkommission an. Entsprechende Modelle gibt es etwa bereits in den Kantonen Freiburg ${ }^{14}, \operatorname{Tessin}^{15}$ oder Wallis ${ }^{16}$. Dieses Gremium sollte idealerweise mehrheitlich mit Fachleuten aus Gerichten, Wissenschaft und Anwaltschaft besetzt sein. Es würde die Bewerbungen nach den vorgenann-

11 Art. 121 Abs. 2 der Verfassung des Kantons Freiburg vom 16. Mai 2004.

12 So auch die Parlamentarische Initiative 20.468, «Stärkung der richterlichen Unabhängigkeit durch Verbot von Mandatssteuern und Parteispenden für Mitglieder der Gerichte des Bundes».

13 Art. 12 des Bundesgesetzes über Bezüge und Infrastruktur der Mitglieder der eidgenössischen Räte und über die Beiträge an die Fraktionen vom 18. März 1988 (Parlamentsressourcengesetz, PRG; SR 171.21).

14 Vgl. dazu Art. 91 ff. des Justizgesetzes vom 31. Mai 2010 (SGF 130.1) und Art. 6 und 27 ff. des Reglements des Justizrats vom 14. Januar 2019 (SGF 130.21) des Kantons Freiburg.

15 Auch im Kanton Tessin werden die Gerichtswahlen durch eine Fachkommission vorbereitet; vgl. dazu Art. 5 ff. Legge sull'organizzazione giudiziaria vom 10. Mai 2006 (RST 177.100) und Regolamento della Commissione d'esperti indipendenti per l'esame e il preavviso delle nuove candidature all'elezione dei magistrati vom 24. September 2019 (RST 177.415).

16 Der Kanton Wallis hat jüngst einen Justizrat eingeführt, dem u.a. die Vorbereitung von Wahlen obliegt; vgl. Art. 46 des Gesetzes über den Justizrat vom 13. September 2019 (SGS 173.7). 
ten Auswahlkriterien prüfen und dem Parlament (oder allenfalls der Gerichtskommission) eine Wahlempfehlung abgeben.

\title{
4. Konkrete Eckpunkte eines Gegenvorschlags
}

\subsection{Amtsdauer: Vorschlag für eine Änderung von Art. 145 BV}

\author{
Art. 145 Amtsdauer \\ ${ }^{1}$ Die Mitglieder des Nationalrates und des Bundesrates sowie die Bundeskanzlerin oder der \\ Bundeskanzler werden auf die Dauer von vier Jahren gewählt. Für die Richterinnen und \\ Richter des Bundesgerichts beträgt die Amtsdauer 6 Jahre. \\ 2 (neu) Richterinnen und Richter des Bundesgerichts werden einmalig bis zur \\ Vollendung des _.. Altersjahrs gewählt; vorbehalten bleibt eine Abberufung aus \\ wichtigem Grund nach Massgabe des Gesetzes.
}

[10] Vorzusehen wäre ausserdem eine Übergangsvorschrift analog dem Vorschlag der JustizInitiative.

\subsection{Auswahlverfahren: Vorschlag für eine Änderung von Art. 168 BV}

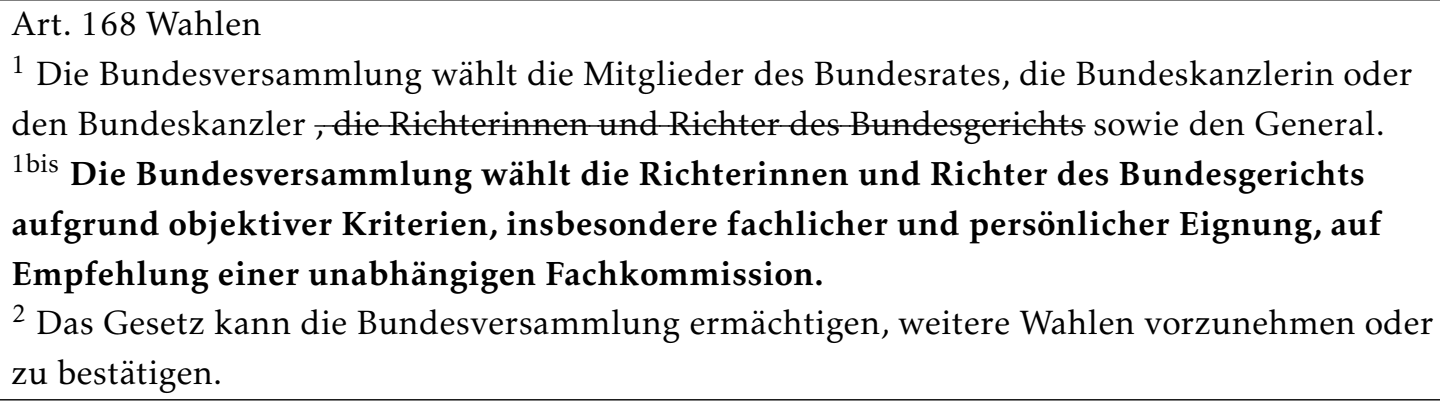

\subsection{Verbot der Mandatssteuer}

[11] Grundsätzlich erscheint das Verbot der Mandatssteuer nicht verfassungswürdig und sollte auf Gesetzesstufe verankert werden. Sollte eine Regelung in der Bundesverfassung als sinnvoll/notwendig erachtet werden, könnte Art. 191c BV wie folgt ergänzt werden:

Art. 191c Richterliche Unabhängigkeit

Die richterlichen Behörden sind in ihrer rechtsprechenden Tätigkeit unabhängig und nur dem Recht verpflichtet.

Ihre Mitglieder dürfen politischen Parteien keine mit dem Amt verbundenen finanziellen Beiträge leisten. 


\subsection{Weitere Hinweise}

[12] Klar ist, dass nicht alle Einzelheiten auf Verfassungsstufe geregelt werden können und sollen. Dies gilt etwa für die Grösse und die Zusammensetzung der Fachkommission, das Abberufungsverfahren oder einen allfälligen finanziellen Ausgleich zufolge des Wegfalls der Mandatssteuern. Hier sind gesetzliche Regelungen bzw. Anpassungen notwendig. Ferner ist zu beachten, dass die Überlegungen zum Bundesgericht auch für die weiteren eidgenössischen Gerichte gleichermassen gelten, weshalb auch insoweit entsprechende Gesetzesanpassungen erforderlich wären. ${ }^{17}$

\section{Ausblick}

[13] Am 5. November 2020 führte die Kommission für Rechtsfragen des Nationalrates (RK-N) eine Anhörung zur Justiz-Initiative durch, zu welcher auch die SVR-ASM eingeladen wurde. Erfreulicherweise fand die SVR-ASM dabei mit den vorstehend dargestellten Lösungsvorschlägen und der Forderung nach einem Gegenentwurf Gehör. Die RK-N nahm, wenn auch mit knapper Mehrheit, einen Antrag für einen indirekten Gegenentwurf an, «der eine objektivere Auswahl der Richterinnen und Richter (Vorselektion durch eine Fachkommission einzig auf der Grundlage der fachlichen und persönlichen Eignung) und eine Prüfung von Alternativen zu den Mandatsabgaben vorsieht sowie die systematische Wiederwahl abschafft und die Abberufung der Bundesrichterinnen und Bundesrichter ermöglicht». ${ }^{18}$

[14] Mit dem Beschluss der RK-N ist ein erster wichtiger Schritt getan. Nun liegt der Ball bei der ständerätlichen Schwesterkommission, die ebenfalls über den Antrag auf einen indirekten Gegenentwurf zu befinden hat. Hoffnungsvoll stimmt in diesem Zusammenhang, dass sich wichtige Exponenten der Kommission wie Ständerat Andrea Caroni, der zugleich die Gerichtskommission präsidiert, bereits öffentlich für einen Gegenentwurf ausgesprochen haben. ${ }^{19}$ Die SVR-ASM ihrerseits wird den parlamentarischen Prozess weiterhin konstruktiv begleiten.

Prof. Dr. iur. Patrick Guidon ist Präsident der Schweizerischen Vereinigung der Richterinnen und Richter (SVR-ASM), Präsident der Strafkammer des Kantonsgerichts St. Gallen und Honorarprofessor für Strafrecht und Strafprozessrecht an der Universität St. Gallen (HSG).

17 Vgl. zu diesen Folgewirkungen auch Andreas Glaser, Die Justiz-Initiative: Besetzung des Bundesgerichts im Losverfahren? Aktuelle Juristische Praxis (AJP) 10/2018, S. 1251 ff., 1260.

18 Vgl. Medienmitteilung der RK-N vom 6. November 2020 (abrufbar unter https://www.parlament.ch/ press-releases/Pages/mm-rk-n-2-2020-11-06.aspx).

19 «Präsident der Gerichtskommission macht Vorschlag für neue Bundesrichterwahlen», Tagblatt vom 13. September 2020 (abrufbar unter: https://www.tagblatt.ch/news-service/inland-schweiz/praesident-der-gerichtskommissionmacht-vorschlag-fuer-neue-bundesrichterwahlen-ld.1256766). 